\title{
The influence of citrus aurantium and caffeine complex versus placebo on the cardiac autonomic response: a double blind crossover design
}

\author{
Brian Kliszczewicz ${ }^{* \dagger} \mathbb{D}$, Emily Bechke ${ }^{\dagger}$, Cassie Williamson, Paul Bailey, Wade Hoffstetter, John McLester
} and Cherilyn McLester

\begin{abstract}
Background: The purpose of this study was to examine the resting cardiac autonomic nervous system's response to the ingestion of a complex containing Citrus aurantium + Caffeine $(C A+C)$ and its influence on recovery following a high-intensity anaerobic exercise bout in habitual caffeine users.

Methods: Ten physically active males $(25.1 \pm 3.9$ years; weight $78.71 \pm 9.53 \mathrm{~kg}$; height $177.2 \pm 4.6 \mathrm{~cm}$; body fat $15.5 \pm 3.13 \%$ ) participated in this study, which consisted of two exhaustive exercise protocols in a randomized crossover design. On each visit the participants consumed either a CA + C (100 mg of CA and $100 \mathrm{mg}$ of C) or placebo (dextrose) capsule. After consumption, participants were monitored throughout a 45-min ingestion period, then completed a repeated Wingate protocol, and were then monitored throughout a 45-min recovery period. Cardiac autonomic function (Heart Rate (HR) and Heart Rate Variability (HRV)) and plasma epinephrine (E) and norepinephrine (NE) were taken at four different time points; Ingestion period: baseline (11), post-ingestion period (12); Recovery period: immediately post-exercise (R1), post-recovery period (R2). Heart rate variability was assessed in 5-min increments.
\end{abstract}

Results: A repeated measures ANOVA revealed significant time-dependent increases in HR, sympathetic related markers of HRV, and plasma $E$ and NE at 12 only in the CA $+C$ trial $(p<0.05)$; however, no meaningful changes in parasympathetic markers of HRV were observed. Participants recovered in a similar time-dependent manner in all markers of HRV and catecholamines following the PLA and CA + C trials.

Conclusion: The consumption of $\mathrm{CA}+\mathrm{C}$ results in an increase of sympathetic activity during resting conditions without influencing parasympathetic activity. CA + C provides no influence over cardiac autonomic recovery.

Keywords: P-synephrine, Heart rate variability, Caffeine, Anaerobic, Recovery

\section{Background}

The cultivation of commercially available supplements has substantially increased throughout recent years, making the use of pharmacologic ergogenic aids more prevalent and readily available to the general population and athletic community. In general, ergogenic aids purport to contain individually unique properties that result in various physiological outcomes (e.g., metabolic); however, a

\footnotetext{
* Correspondence: Bkliszcz@kennesaw.edu

${ }^{\dagger}$ Brian Kliszczewicz and Emily Bechke contributed equally to this work. Department of Exercise Science and Sport Management, Kennesaw State University, Kennesaw, GA, USA
}

majority of these claims are overstated or not fully understood. Recently, a growing interest in the combined supplementation of Citrus aurantium (CA) and Caffeine (C) has emerged due to its ability to decrease rate of fatigue during exercise and increase metabolic rate $[1,2]$.

While examining these supplements individually, $C$ has been known to stimulate the central nervous system (CNS) (i.e. increase heart rate, cognitive function, blood pressure) which in turn increases motor function, availability of plasma free fatty acids, and increased performance outcomes during endurance exercise [3-5]. Whereas CA is most popularly used as a weight loss supplement, 
which is attributed to its primary protoalkaloidal constituent, p-synephrine [6-8]. P-synephrine has an affinity to $B-3$ receptors and therefore enhances lipolysis and thermogenesis. CA was previously theorized to produce similar effects on the CNS as ephedra due to p-synephrine's similarities in chemical structure. However, it has recently been found that the influence of CA over CNS appears to be minimal, although not fully understood [6]. To this point, the literature is conflicting in regards to the magnitude of the effect of CA on the cardiovascular system, especially when combined with $C[7,9,10]$.

A study conducted by Ratamess et al., [2] supports the notion that when combined, caffeine $(100 \mathrm{mg})$ and p-synephrine $(100 \mathrm{mg})$ can increase local muscle endurance within multiple sets during resistance training. The findings of this study are suggestive of enhanced recovery between multiple sets [2], which may therefore translate to the recovery of other mechanisms of physiological stress and control. The analysis of the autonomic nervous system (ANS) is a viable method to assess transient alterations and stress on the body, and may also provide insight into systemic readiness [11]. The homeostatic condition of the body is maintained through the sensitivity and the responsiveness of the ANS to internal and external stimuli $[11,12]$. Fluctuations of the ANS are observed through changes in its two branches; the sympathetic nervous system (SNS) and parasympathetic nervous system (PNS). ANS activity can be indirectly measured through the observation of cardiac autonomic activity (e.g., vagal and sympathetic modulations), which can be measured through heart rate variability (HRV). In conjunction with HRV measures, plasma catecholamines, epinephrine $(\mathrm{E})$ and norepinephrine (NE), provide direct markers of SNS activity and allow for a more holistic view of ANS function.

To the authors' knowledge, no known research has been conducted examining the influence of $\mathrm{CA}+\mathrm{C}$ complex supplementation on cardiac autonomic activity and recovery following a high-intensity exhaustive exercise protocol. Improvements in acute ANS recovery may translate to reduce transient stresses within the CV system as well as prevent systemic over reaching $[11,13,14]$. Therefore, the purpose of this study was to examine the resting ANS response to the ingestion of $\mathrm{CA}+\mathrm{C}$ as well as its influence on recovery following an exhaustive anaerobic exercise protocol.

\section{Methods}

\section{Participants}

Fourteen apparently healthy males who habitually consume caffeine (95-300 mg serving per day, at least 4 days a week) were recruited for this study. Prior to participation, all individuals were made aware of the procedures and risks associated with the study and signed an informed consent. A health history questionnaire (HHQ) and physical activity readiness questionnaire (PAR-Q) were administered in order to ensure that participants were capable of engaging in vigorous physical activity without physician's clearance as defined by the guidelines provided by the American College of Sports Medicine [15]. Any individual who reported having orthopedic conditions, cardiovascular, pulmonary, or metabolic disease were excluded from the study. Those who regularly over consumed caffeine $(\geq 300 \mathrm{mg} /$ day $)$ were also excluded from the study.

Physical activity inclusion criteria required all participants to engage in at least three-days of aerobic training and two-days of resistance training per week for the previous six months. Participants were recruited via word of mouth from the local metropolitan area. Prior to all sessions, participants were asked to wear light and comfortable clothing, fast for a minimum of four-hours, avoid exercise for $24 \mathrm{~h}$, and avoid caffeine consumption for $12 \mathrm{~h}$. The Institutional Review Board approved all testing procedures and protocols prior to beginning data collection.

\section{Experimental design}

The study was performed in a double-blind, placebocontrolled, randomized crossover fashion in which only one investigator knew the contents of the supplementation; this investigator was not involved in the collection or analysis of the study outcome measures. Participants were asked to attend two separate sessions in the exercise physiology laboratory, with both visits occurring within a nine-day period and a minimum of 72-h between visits. All visits were performed between 5:00 am 7:00 am. The first visit consisted of obtaining informed consent, PAR-Q, HHQ, and anthropometric measures. Height $(\mathrm{cm})$ and weight $(\mathrm{kg})$ were collected using an electronic physicians scale (Tanita WB 3000, Arlington Heights, IL) and body fat percent (BF\%) was collected via a dual-energy $x$-ray absorptiometry scan (GE Lunar iDXA, Madison, WI).

The remaining two visits can be described in two overarching sections; the ingestion period with pre and post time points (I1 \& I2) and the recovery period with pre and post time points (R1 \& R2). The ingestion period consisted of baseline measures (I1), which included veinipuncture and the fitting of a polar heart rate monitor, followed by the consumption of either the supplement $(\mathrm{CA}+\mathrm{C})$ or placebo (PLA). The 45-min ingestion period was initiated after the participants consumed $\mathrm{CA}+\mathrm{C}$ or PLA. Upon the completion of the ingestion period, a post-ingestion venipuncture were performed (I2). Participants then performed a standardized warm-up prior to initiating the anaerobic exhaustive exercise protocol. Immediately following the exercise protocol a post exercise venipuncture was performed (R1). Then, participants 
were monitored throughout a 45-min recovery period. At the end of this recovery period the final venipuncture was taken (R2). Cardiac activity was continuously recorded during the 45-min ingestion and recovery periods. Analysis of these recordings were made in 5-min segments beginning at the 5-10th minutes of the ingestion and recovery periods. These values are present as time points I1 \& R1 (respectively). Additionally, the 40-45th minutes of the ingestion and recovery periods and are present as time points I2 \& R2 (respectively). The study design can be seen in Fig. 1.

\section{Exhaustive exercise protocol}

Upon the completion of the 45-min ingestion period, participants were allotted a seven-and-a-half minute warm up on a Monark ergometer (Monark 828E Ergomedic Test Cycle, Vansbro, Sweden) while pedaling between 50 and $100 \mathrm{rpm}$ at a resistance of $1.5 \mathrm{kp}$. Participants were immediately walked to an electronically braked cycle ergometer (Sport Excalibur, Lode BV, Groningen, The Netherlands), where the bike was adjusted to the appropriate settings in order to ensure the knee was at a slight bend at the bottom of the revolution. Bike settings were repeated for both trials. Following the appropriate adjustments, participants feet were strapped into the pedals and the protocol was initiated. The start of the exhaustive exercise protocol comprised of a one-minute warm-up period performed at $50 \mathrm{~W}$ with a rolling start into the Wingate test. Each Wingate test was 30-s in duration and participants were encouraged to pedal at their maximal effort against a resistance of $0.80 \mathrm{Nm} / \mathrm{kg}$ [16]. There was a total of three Wingate tests performed with a two-minute active recovery period between each test. The active recovery was a self-selected pedal rate against a resistance of $50 \mathrm{~W}$ and a rolling start into the subsequent Wingate test. At the completion of the last Wingate test, participants were walked to a separate room to undergo a post exercise venipuncture and to begin the measurements of cardiac autonomic recovery measures (R1-R2). Pre-testing protocols on the electronically braked cycle ergometer followed manufacturer guidelines.

\section{Blood collection and analysis}

A trained phlebotomist drew six milliliters $(\mathrm{ml})$ of blood via the antecubital vein during four-time periods throughout the study: I1, I2, R1, R2 (Fig. 1). Blood draws were collected in lithium heparin tubes and inverted based on the manufactures' recommendations prior to centrifugation. Samples were centrifuged at $2500 \mathrm{rpm}$ for 15 -min, then aliquoted and stored in a $-80{ }^{\circ} \mathrm{C}$ freezer until subsequent assay analysis. Plasma samples were assayed for E and NE using commercially available ELISA kits (Abnova, Taoyuan City, Taiwan). In order to account for the plasma volume shifts following the exercise bout, all samples were normalized by using the established protocols of Dill and Costill [17]. Hematocrit (Hct) and hemoglobin $(\mathrm{Hb})$ were collected via finger sticks at each venipuncture time point (Alere Hemopoint 2).

\section{Heart rate variability collection and analysis}

Heart Rate Variability is a non-invasive measurement that quantifies the timing between consecutive R-R intervals. The measurements are derived from an electrocardiogram or HR detection device (i.e. HR monitors) [18]. Heart Rate Variability and HR recordings were collected using the Polar monitor system and transferred to the Polar Team ${ }^{2}$ software (Lake Success, NY). Heart rate monitors were positioned under the sternum against bare skin. Throughout each 45 -min recording period, participants were seated in a quiet, dimly lit room with no external stimuli.

Analysis was completed through the online Kubios Software (Kubios V 2.2, Joensuu, Finland) wherein recordings were transformed into a tachogram, which plots the successive $R-R$ intervals (y-axis) against the number of beats within the time series (x-axis). Heart Rate Variability markers were analyzed in five-minute segments during the beginning (5-10 min: I1, R1) and end (40-45 min: I2,

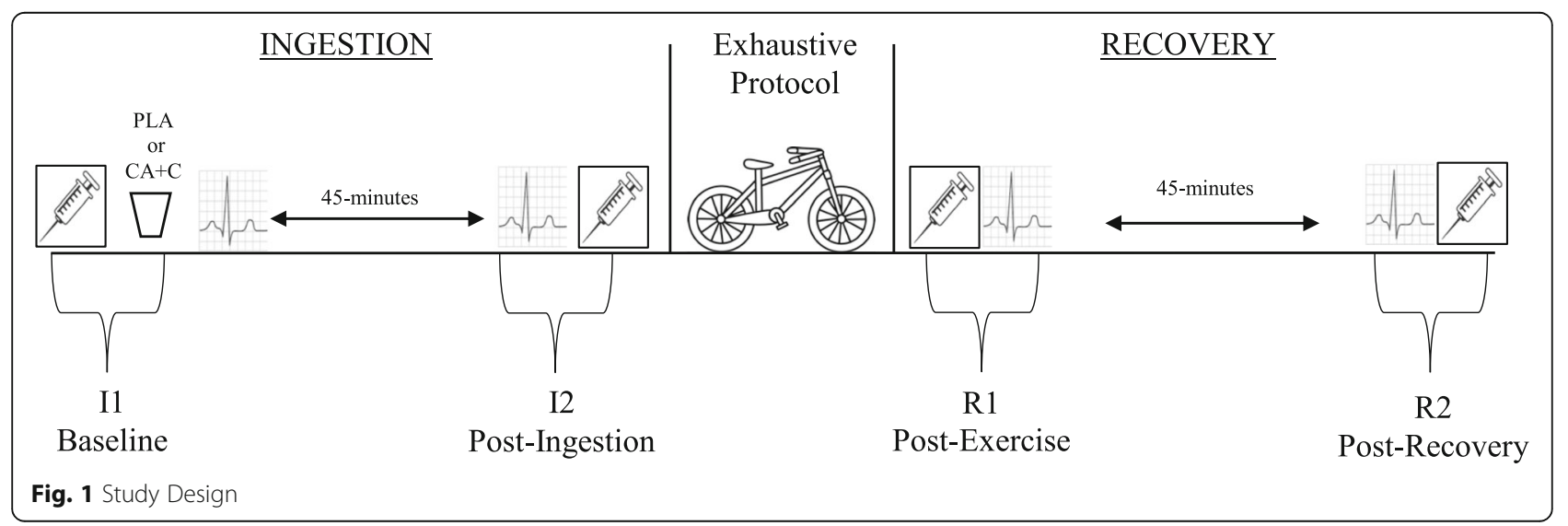


R2) of the ingestion and the recovery periods. During analysis, an artifact correction of "low" with a sensitivity set to identify any R-R abnormality $\pm 0.35 \mathrm{~s}$ was applied using a piecewise cubic spline interpolation method in order to filter additional artifact noise present in the tachogram (Kubios V 2.2, Joensuu, Findland) [19, 20]. Any segments that contained three or more irregular R-R intervals were excluded from analysis.

The markers chosen for this study were the time domain indexes of the root mean square of successive differences (RMSSD) and the standard deviation of normal-to-normal intervals (SDNN); the frequency domain measures of High Frequency Power (HF) $(0.15-0.40 \mathrm{~Hz})$, normalized High Frequency Power (HFnu), Low Frequency Power (LF) $(0.04-0.15 \mathrm{~Hz})$, normalized Low Frequency Power (LFnu), and their ratio LF/HF. The Fast Fourier Transformation was applied to the frequency domain makers. Frequency domain measures come with inherent limitations related to ANS interpretation due to sensitivities to breathing frequencies and therefore will be assessed along with time domain measures [21]. RMSSD and $\mathrm{HF}$ are widely recognized as markers of vagal activity $[18,22]$, while SDNN and LFnu are believed to provide insight into SNS influence, though they possess activity from the PNS [23, 24]. LF/HF ratio provides insight into ANS balance [25]. All R-R interval recordings were measured using a window width of $256 \mathrm{~s}$ and overlap of $50 \%$ through the specialized HRV software (Kubios V 2.2, Joensuu, Findland).

\section{Supplement preparation}

$\mathrm{CA}$ and $\mathrm{C}$ powder were purchased from Blackburn distributions (Caffeine powder, Blackburn distributions limited, Nelson Lancashire, England; Citrus aurantium powder, Blackburn distributions limited, Nelson Lancashire, England). The PLA contained $200 \mathrm{mg}$ of dextrose, whereas the supplement contained a combination of CA $(100 \mathrm{mg})$ and $\mathrm{C}(100 \mathrm{mg})$. Each component was measured using an electronic supplement scale and encapsulated in green, non-translucent, size zero gelatin capsules. The identity of the content within the capsules was not revealed until all data were collected and statistical analyses were completed.

\section{Statistical analysis}

All data were analyzed using the statistical software package SPSS (SPSS, Version 24 for Mac, Chicago, IL). A Shapiro-Wilk test was performed to examine the normality of distribution on the HRV markers: RMSSD and SDNN. In order to assess changes in time within trial (CA $+\mathrm{C} / \mathrm{PLA}$ ) repeated measures analysis of variance (ANOVA) were run (Ingestion: I1 vs. I2; Recovery: R1 vs. R2) in cardiac autonomic markers (HRV and HR) and plasma catecholamines (E and NE). Significance for all statistical analyses was set at $\leq 0.05$. The data is presented as the mean \pm standard deviation (SD). In order to determine the effect size, the recommended guidelines of Quintana were used. [26]. Thresholds for effect size were the following; a small $(<0.25)$ moderate $(0.50)$, and large effect $(0.90)$.

\section{Results}

Four participants were removed from the study due to adverse reactions to the phlebotomy procedure (i.e. vaso-vagal reactions) $(n=2)$, pain brought on by brochiospasm $(n=1)$, and the presence of more than three irregular R-R intervals in the recordings $(n=1)$. Therefore, a total of ten physically active males completed the study. Participant characteristics can be seen in Table 1. Additionally, normality was violated in several HRV markers and therefore the natural logarithmic transformation (ln) was applied prior to further statistical analysis: RMSSD (lnRMSSD), SDNN (lnSDNN), HF (lnHF), LF(lnLF).

During the PLA ingestion phase, no significant $(p>0.05)$ time effects were found in any cardiac autonomic markers or catecholamines except for a significant decrease in HFnu and a significant increase in LFnu $(p<0.05)$. However, during the $\mathrm{CA}+\mathrm{C}$ trial ingestion period, a significant time effect $(\mathrm{p}<0.05)$ was observed from I1 to I2 with increases in $\mathrm{HR}, \operatorname{lnSDNN}$, LFnu, NE, E, and a decrease in HFnu. While examining the recovery period in the PLA and CA + $C$ trials, no significant time effects occurred in HFnu, LFnu, or LF/HF. A significant decrease in HR, InRMSSD, and $\operatorname{lnSDNN}$ occurred along with a significant decrease in $\mathrm{E}$ and NE. Means \pm SD and effect size (Cohen's d) can be seen for the ingestion period in Table $2 \mathrm{~A}$ and the recovery period in Table 2B.

\section{Discussion}

The purpose of this study was to examine the resting ANS response to the ingestion (I1 - I2) of CA + C as well as its influence on the ANS response to an exhaustive exercise protocol (R1 - R2). During the ingestion period HR, lnLF, LFnu, E and NE significantly increased in the CA + C trial, indicating an enhanced sympathetic response to the supplementation. Interestingly, a significant decrease in HFnu was observed while no changes in lnHF or lnRMSSD occurred despite a significant increase in $\ln S D N N$. All cardiac autonomic markers demonstrated a time-dependent shift towards baseline following the exercise protocol

Table 1 Participant Characteristics $(N=10)$

\begin{tabular}{ll}
\hline Characteristic & Mean \pm SD \\
\hline Age $(\mathrm{y})$ & $25.1 \pm 3.8$ \\
Height $(\mathrm{cm})$ & $177.2 \pm 4.6$ \\
Weight $(\mathrm{kg})$ & $78.8 \pm 9.4$ \\
Body Fat (\%) & $15.5 \pm 3.0$ \\
Caffeine/day (mg) & $209 \pm 95.5$ \\
\hline
\end{tabular}


Table 2 Markers of ANS activity during the Ingestion (A) and Recovery Periods (B)

\begin{tabular}{|c|c|c|c|c|c|c|c|c|}
\hline \multirow[t]{2}{*}{ A. Ingestion Period } & \multicolumn{4}{|l|}{ PLA } & \multicolumn{4}{|l|}{$C A+C$} \\
\hline & 11 & 12 & $p$-value & Cohen's d & 11 & 12 & $p$-value & Cohen's d \\
\hline Heart Rate (bpm) & $62.6 \pm 14.34$ & $65.5 \pm 10.96$ & 0.60 & 0.23 & $59.7 \pm 7.93$ & $64.6 \pm 9.13$ & 0.04 & 0.57 \\
\hline InRMSSD (ms) & $3.95 \pm 0.49$ & $3.87 \pm 0.40$ & 0.57 & 0.17 & $4.0 \pm 0.50$ & $4.08 \pm 0.45$ & 0.45 & 0.16 \\
\hline InSDNN (ms) & $4.32 \pm 4.40$ & $4.40 \pm 0.33$ & 0.59 & 0.22 & $4.36 \pm 0.48$ & $5.04 \pm 0.56$ & 0.03 & 1.28 \\
\hline $\operatorname{lnHF}\left(\mathrm{ms}^{2}\right)$ & $6.65 \pm 1.00$ & $6.55 \pm 0.85$ & 0.70 & 0.12 & $6.95 \pm 0.79$ & $7.04 \pm 0.82$ & 0.70 & 0.11 \\
\hline HFnu & $42.92 \pm 16.87$ & $29.37 \pm 16.06$ & 0.01 & 0.82 & $39.01 \pm 17.13$ & $31.78 \pm 12.49$ & 0.03 & 0.48 \\
\hline $\operatorname{lnLF}\left(m s^{2}\right)$ & $7.00 \pm 1.00$ & $7.51 \pm 0.73$ & 0.21 & 0.58 & $7.43 \pm 1.05$ & $7.86 \pm 0.71$ & 0.05 & 0.48 \\
\hline LFnu & $57.01 \pm 16.89$ & $70.62 \pm 16.07$ & 0.01 & 0.82 & $58.95 \pm 20.45$ & $68.20 \pm 12.50$ & 0.03 & 0.55 \\
\hline $\mathrm{LF} / \mathrm{HF}$ & $2.08 \pm 2.29$ & $3.20 \pm 1.54$ & 0.07 & 0.57 & $2.40 \pm 2.31$ & $2.75 \pm 1.63$ & 0.45 & 0.18 \\
\hline $\mathrm{E}(\mathrm{nmol} / \mathrm{L})$ & $4.40 \pm 2.93$ & $3.97 \pm 2.05$ & 0.41 & 0.17 & $3.53 \pm 2.08$ & $4.96 \pm 2.84$ & 0.02 & 0.58 \\
\hline $\mathrm{NE}(\mathrm{nmol} / \mathrm{L})$ & $21.56 \pm 5.57$ & $23.40 \pm 9.63$ & 0.51 & 0.23 & $18.16 \pm 3.91$ & $28.29 \pm 7.29$ & 0.00 & 1.73 \\
\hline \multirow[t]{2}{*}{ B. Recovery Period } & \multicolumn{4}{|l|}{ PLA } & \multicolumn{4}{|l|}{$C A+C$} \\
\hline & R1 & R2 & $p$-value & Cohen's d & R1 & R2 & $p$-value & Cohen's d \\
\hline Heart Rate (bpm) & $102.56 \pm 10.79$ & $87.6 \pm 11.30$ & 0.00 & 1.35 & $111.56 \pm 11.37$ & $94.4 \pm 9.77$ & 0.00 & 1.35 \\
\hline InRMSSD (ms) & $1.42 \pm 0.49$ & $2.54 \pm 0.66$ & 0.00 & 1.90 & $1.51 \pm 0.84$ & $2.50 \pm 0.68$ & 0.03 & 1.29 \\
\hline InSDNN (ms) & $2.90 \pm 0.31$ & $3.57 \pm 0.49$ & 0.01 & 1.62 & $2.89 \pm 0.45$ & $3.71 \pm 0.58$ & 0.01 & 1.59 \\
\hline $\operatorname{lnHF}\left(\mathrm{ms}^{2}\right)$ & $1.59 \pm 0.62$ & $3.92 \pm 1.30$ & 0.00 & 2.30 & $1.50 \pm 1.50$ & $4.23 \pm 1.22$ & 0.00 & 2.01 \\
\hline HFnu & $12.33 \pm 7.30$ & $13.61 \pm 11.11$ & 0.78 & 0.14 & $19.37 \pm 20.31$ & $18.00 \pm 13.06$ & 0.71 & 0.08 \\
\hline $\operatorname{lnLF}\left(\mathrm{ms}^{2}\right)$ & $3.69 \pm 0.97$ & $6.04 \pm 1.26$ & 0.00 & 2.09 & $3.19 \pm 1.12$ & $5.71 \pm 1.50$ & 0.00 & 1.90 \\
\hline LFnu & $87.62 \pm 7.27$ & $86.37 \pm 11.14$ & 0.78 & 0.13 & $80.45 \pm 20.50$ & $81.95 \pm 13.11$ & 0.69 & 0.09 \\
\hline $\mathrm{LF} / \mathrm{HF}$ & $9.69 \pm 4.83$ & $12.18 \pm 12.00$ & 0.47 & 0.27 & $7.63 \pm 4.22$ & $7.18 \pm 5.15$ & 0.82 & 0.09 \\
\hline $\mathrm{E}(\mathrm{nmol} / \mathrm{L})$ & $31.50 \pm 19.09$ & $4.22 \pm 2.91$ & 0.00 & 2.00 & $38.24 \pm 26.44$ & $5.50 \pm 2.34$ & 0.00 & 1.74 \\
\hline $\mathrm{NE}(\mathrm{nmol} / \mathrm{L})$ & $158.20 \pm 82.26$ & $30.11 \pm 14.08$ & 0.00 & 2.17 & $179.14 \pm 61.37$ & $35.20 \pm 8.51$ & 0.00 & 3.2 \\
\hline
\end{tabular}

Chronotropic markers of ANS activity is represented as HR; Markers of Heart Rate Variability (HRV) are the log transformed mean square of successive N-N intervals (InRMSSD), standard deviation of the N-N intervals (InSDNN), the High Frequency (InHF), Low Frequency (InLF) and the ratio (LF/HF); Plasma Catecholamines are represented as Epinephrine (E) and Norepinephrine (NE). All data are presented as Means \pm SD

$\leq 0.05$ significant time effects

except for LFnu, HFnu, and LF/HF. Further points of consideration are provided below.

\section{Ingestion period}

Resting HR is a primary marker in cardiac autonomic activity and is affected through several intrinsic and extrinsic factors; however, the influence of the combination of $\mathrm{CA}+\mathrm{C}$ on resting HR is relatively unknown. The limited amount of information available pertains to the isolated components $\mathrm{CA}$ and $\mathrm{C}$, with only one known study to have examined the combination of both [2]. For instance, Min et al., [10] examined only p-synephrine, the active component of $\mathrm{CA}$, and found no changes in resting HR at one-hour, three-hours, six-hours, or eight-hours following consumption. Furthermore, recent studies have shown little no changes in resting HR with caffeine consumption alone in habitual caffeine consumers [27, 28]. When combining a $100 \mathrm{mg}$ of CA, and $100 \mathrm{mg}$ of C, Ratamess et al. [2] observed no significant changes in resting HR following a three-week washout period from caffeine when compared to controls. In contrast, the findings of the current study demonstrated a time-dependent increase in HR following the consumption of the $\mathrm{CA}+\mathrm{C}$ complex. No time-dependent changes were observed during the ingestion phase for the PLA trial. This discrepancy may be due to differences in the experimental design. For instance, the participants in the Ratamess et al. [2] study underwent a three day exposure to the $\mathrm{CA}+\mathrm{C}$ complex prior to testing, while in the current study participants were not given the $\mathrm{CA}+\mathrm{C}$ complex prior to the experimental trials, which may account for the difference in the response.

Overall, the evaluation of $\mathrm{CA}$ or $\mathrm{CA}+\mathrm{C}$ on markers of ANS activity is currently understudied, but information regarding other stimulants (e.g. caffeine) is replete $[3-5,29,30]$. For instance, Rauh et al. [31] found that the consumption of either $100 \mathrm{mg}$ or $200 \mathrm{mg}$ of caffeine in habitual users failed to alter any examined marker of HRV. Zimmerman et al. [28] examined caffeine consumption in both habitual and non-habitual consumers and found no effect on SNS related activity (LF and LF/HF), but enhanced PNS activity (RMSSD and HF) in habitual users was observed. Conversely, Yoshinaga et al., observed 
significant increases in the power spectral density in both LF and HF following the ingestion of $4 \mathrm{mg} \cdot \mathrm{kg}$ of bodyweight [32]. The findings of the current study provide equivocal results to the literature, where the consumption of $\mathrm{CA}+\mathrm{C}$ increased HR, lnLF, LFnu, E, and NE; indicating an increase in SNS activity while showing no changes in PNS markers (lnRMSSD and lnHF) during the ingestion period. Interestingly, a nonsignificant rise of $\operatorname{lnLF}$ and reduction of lnHF was observed following the PLA trial. However, when evaluating LFnu and HFnu a similar yet significant changed was observed, demonstrating a relative change in the ratios rather than the absolute values of the power spectral density. This may in part be due to the anticipation of the upcoming exhaustive protocol and pre-performance anxiety, resulting in minor shifts of ANS activity. Future studies should evaluate and account for pre trial emotional stress.

When evaluating plasma markers of SNS activity, it has been proposed that circulating sympathetic biomarkers $E$ and NE increase following consumption of caffeine [33, 34]; however, a recent study demonstrated caffeine to have little to no influence over resting values [35]. The observed changes in $\mathrm{E}$ and NE within the current study support the previous notion that caffeine influences resting plasma levels and reflect the changes observed in SNS related HRV markers following the CA $+C$ ingestion. The lack of change in the lnRMSSD and lnHF in the presence of increased SNS activity acts against the traditional interplay between PNS and SNS balance. Generally, with increases in SNS activity a withdrawal of vagal tone occurs. However, this was not observed and could be the result of a decreased sensitivity to caffeine or the rested state of the participant, which resulted in the attenuation of vagal activity.

Regardless of the mechanisms involved, the overall cardiac autonomic response observed during the ingestion phase is suggestive of a "priming" of the SNS response to the CA $+\mathrm{C}$ supplementation. Traditionally, it is believed that ANS activity is balanced between the PNS and SNS branches, exhibiting an inverse relationship [12]. However, the PNS/SNS interplay appears to be more complex, and likely exhibits their influences upon each other on a spectrum, rather than direct counter balance. The findings of this study are indicative of this type of relationship between the SNS and PNS branches, specifically the lack of change in resting PNS activity when compared to the time-based increases in SNS drive observed in the $\mathrm{CA}+\mathrm{C}$ trial. Specifically, there was an increase in $\operatorname{lnSDNN}$ and $\operatorname{lnLF}$ without the presence of altered vagal activity. This is important because these markers are believed to have influences stemming from both the SNS and PNS [36]. Therefore, with no discernable changes within markers of vagal activity, it can be inferred that the increases of $\operatorname{lnLF}$ and $\operatorname{lnSDNN}$ are the result of changes seen in SNS activity. This response adds to the understanding of the level of complexity within ANS control and should be further investigated to determine thresholds between the various markers.

\section{Recovery period}

Following the exhaustive protocols, HR was significantly elevated in both trials and recovered in a similar time-dependent fashion (Table 2A and B). This is consistent with the findings of Haller et al. [37] who did not observe any differences in post-exercise recovery of HR when comparing a commercially available supplement (21 $\mathrm{mg}$ of synephrine, $303.8 \mathrm{mg}$ of caffeine, and various additives) to a placebo during the 30-min to 12-h following a moderate exercise bout [37]. Additionally, markers of HRV following the exhaustive protocol demonstrated nearly identical physiological responses, with a decrease in activity post exercise and a gradual increase toward baseline values, which is a commonly observed post exercise response [11, 38]. A similar yet inverse response was observed in circulating plasma $\mathrm{E}$ and NE, with substantial increases post exercise and a return to baseline values within 45-min post (R2). It is important to note that though increased SNS activity was observed following the ingestion of $\mathrm{CA}+\mathrm{C}$, the effect was lost following the exhaustive protocol. Therefore, the findings are suggestive that the influence of $\mathrm{CA}+\mathrm{C}$ occurs only in a resting environment and does not influence the recovery of the system.

Though it was outside the scope of the current study to establish mechanisms involved with $\mathrm{CA}+\mathrm{C}$ consumption, we will postulate on potential factors involved in the observed findings. The ingestion phase (I1 - I2) at rest was the only period in which $\mathrm{CA}+\mathrm{C}$ appeared to have an effect on SNS activity. As previously mentioned, recent studies have demonstrated that $\mathrm{C}$ provides little cardiovascular stimulation and more so acts to improve PNS activity rather than inhibit in habitual users [28]. However, it should not be overlooked that the research is conflicting in habitual consumers and that $\mathrm{C}$ has been shown to alter SNS activity through increased sensitivity to circulating E and NE [29]. Within the current study, plasma $\mathrm{E}$ and $\mathrm{NE}$ significantly increased following $\mathrm{CA}+\mathrm{C}$ ingestion in our habitual $C$ population $(209 \pm 95.5 \mathrm{mg} /$ day $)$. There was no withdrawal of PNS activity despite a significant increase in resting HR, which could be explained by the combined effects of circulating $\mathrm{E}$ and $\mathrm{NE}$ as well as improved sensitivity related to $C$. The acting ingredient of $\mathrm{CA}, \mathrm{p}$-synephrine, works primarily on the $\mathrm{B}-3$ receptors on adipose tissue and therefore is unlikely to have any direct impact on autonomic function [7]. Indirectly, the increased activity of lipolysis, and thermogenesis caused by p-synephrine could have elevated SNS activity, which was demonstrated by Reimann et al. [39] who found that acute hyperlipidemia increased both HR and sympathetic 
drive. Though we did not measure changes in plasma lipids we can postulate that the known action of p-synephrine could have elevated plasma levels and consequently influenced sympathetic activity.

\section{Conclusion}

In summary, the $\mathrm{CA}+\mathrm{C}$ complex (100 mg each) used in this study was enough to elicit what can best be described as a priming of the SNS response after consumption. As such, the physiological aspects involved with $\mathrm{CA}+\mathrm{C}$ supplementation appear to have the greatest influence in a resting environment. Beyond modest increases in SNS activity at rest, little benefit was observed during the exhaustive protocol recovery period, which was the primary purpose of the investigation. Therefore, the authors conclude that in healthy male participants who regularly consume caffeine, $\mathrm{CA}+\mathrm{C}$ does not improve recovery of cardiac autonomic function following an exhaustive exercise protocol. The observed priming of the SNS activity with no alterations of PNS activity provided new insight into the complex relationship of the ANS and warrants further investigation.

\section{Abbreviations}

ANS: Autonomic nervous system; Body Fat: BF\%; C: Caffeine; CA + C: Caffeine and Citrus aurantium Complex; CA: Citrus aurantium; CNS: Central nervous system; E: Epinephrine; HCT: Hematocrit; HF: High frequency; HFnu: High frequency normalized; $\mathrm{HHQ}$ : Health history questionnaire; HR: Heart rate; HRV: Heart rate variability; LF: Low frequency; LF/HF: Low frequency/ High frequency ratio; LFnu: Low frequency normalized; NE: Norepinephrine; PARQ: Physical activity readiness questionnaire; PLA: Placebo;

PNS: Parasympathetic nervous system; RMSSD: Root mean square of successive R-R intervals; SDNN: Standard deviation of N-N intervals; SNS: Sympathetic nervous system

\section{Acknowledgements}

Not applicable.

\section{Funding}

Not Applicable.

\section{Availability of data and materials}

The data sets used during the current study are available from the corresponding author upon reasonable request.

\begin{abstract}
Authors' contributions
BK contributed to study design, data collection (HRV and Biomarker), data analysis, major contribution to the writing of the manuscript. EB contributed to data collection, performed HRV analysis and interpretation, blood assay analysis, conducted literature review, and major contribution to the writing of the manuscript. CW contributed with data collection, assisted with data analysis (Biomarker), and moderate contributions to the editing of the manuscript. PB contributed to study design, data collection, moderate editing of the manuscript. WH significant contribution to data collection, moderate editing of the manuscript. JM contributed to study design, data statistical analysis, and moderate editing of manuscript. CM contributed to the study design, data collection, moderate editing of manuscript, and procurement of funds. All authors read and approved the final manuscript.
\end{abstract}

\section{Ethics approval and consent to participate}

The Institutional Review Board approved all testing procedures and protocols prior to beginning data collection (17-220) Participants read and sign an informed consent prior too participating in this study.

\section{Consent for publication}

Not Applicable.

\section{Competing interests}

These authors declare that they have no competing interest and have no relation too the supplement or associated companies.

\section{Publisher's Note}

Springer Nature remains neutral with regard to jurisdictional claims in published maps and institutional affiliations.

Received: 1 February 2018 Accepted: 16 July 2018

Published online: 24 July 2018

\section{References}

1. Colker CM, Kaiman DS, Torina GC, Perlis T, Street C. Effects of Citrus aurantium extract, caffeine, and St. John's wort on body fat loss, lipid levels, and mood states in overweight healthy adults. Curr Ther Res. 1999;60(3):145-53.

2. Ratamess NA, Bush JA, Kang J, Kraemer WJ, Stohs SJ, Nocera VG, Leise MD, Diamond KB, Campbell SC, Miller HB, et al. The effects of supplementation with p-Synephrine alone and in combination with caffeine on metabolic, Lipolytic, and cardiovascular responses during resistance exercise. J Am Coll Nutr. 2016;35(8):657-69.

3. HOGERVORST E, BANDELOW S, SCHMITT J, JENTJENS R, OLIVEIRA M, ALLGROVE J, CARTER T, GLEESON M. Caffeine improves physical and cognitive performance during exhaustive exercise. Med Sci Sports Exerc. 2008:40(10):1841-51

4. Ganio MS, Klau JF, Casa DJ, Armstrong LE, Maresh CM. Effect of caffeine on sport-specific endurance performance: a systematic review. J Strength Cond Res. 2009;23(1):315-24.

5. Spriet L, MacLean D, Dyck D, Hultman E, Cederblad G, Graham T. Caffeine ingestion and muscle metabolism during prolonged exercise in humans. Am J Physiol Endocrinol Metab. 1992;262(6):E891-8.

6. Stohs SJ, Preuss HG, Shara M. The safety of Citrus aurantium (bitter orange) and its primary protoalkaloid p-synephrine. Phytother Res. 2011;25(10):1421-8.

7. Stohs SJ, Preuss HG, Shara M. A review of the receptor-binding properties of p-synephrine as related to its pharmacological effects. Oxid Med Cell Longev. 2011;2011:482973.

8. Stohs SJ, Preuss HG, Shara M. A review of the human clinical studies involving Citrus aurantium (bitter orange) extract and its primary protoalkaloid p-synephrine. Int J Med Sci. 2012;9(7):527.

9. Bui LT, Nguyen DT, Ambrose PJ. Blood pressure and heart rate effects following a single dose of bitter orange. Ann Pharmacother. 2006;40(1):53-7.

10. Min B, Cios D, Kluger J, White CM. Absence of QTc-interval-prolonging or hemodynamic effects of a single dose of bitter-orange extract in healthy subjects. Pharmacotherapy. 2005;25(12):1719-24.

11. Stanley J, Peake JM, Buchheit M. Cardiac parasympathetic reactivation following exercise: implications for training prescription. Sports Med. 2013; 43(12):1259-77.

12. Borresen J, Lambert MI. Autonomic control of heart rate during and after exercise : measurements and implications for monitoring training status. Sports Med. 2008;38(8):633-46.

13. Eijsvogels TM, George KP, Thompson PD. Cardiovascular benefits and risks across the physical activity continuum. Curr Opin Cardiol. 2016;31(5):566-71

14. Fry AC, Kraemer WJ, Van Borselen F, Lynch JM, Triplett NT, Koziris LP, Fleck SJ. Catecholamine responses to short-term high-intensity resistance exercise overtraining. J Appl Physiol (1985). 1994;77(2):941-6.

15. Medicine ACOS: ACSM's guidelines for exercise testing and prescription, 10 edn: Lippincott Williams \& Wilkins; 2017.

16. Maclntosh BR, Rishaug P, Svedahl K. Assessment of peak power and shortterm work capacity. Eur J Appl Physiol. 2003;88(6):572-9.

17. Dill DB, Costill DL. Calculation of percentage changes in volumes of blood, plasma, and red cells in dehydration. J Appl Physiol. 1974;37(2):247-8.

18. Camm AJ, Malik M, Bigger J, Breithardt G, Cerutti S, Cohen RJ, Coumel P, Fallen EL, Kennedy HL, Kleiger RE. Heart rate variability: standards of measurement, physiological interpretation and clinical use. Task force of the European Society of Cardiology and the North American Society of Pacing and Electrophysiology. Circulation. 1996;93(5):1043-65.

19. Nakamura FY, Pereira LA, Cal Abad CC, Cruz IF, Flatt AA, Esco MR, Loturco I. Adequacy of the ultra-short-term HRV to assess adaptive processes in youth female basketball players. J Hum Kinet. 2017;56:73-80. 
20. Tarvainen MP, Niskanen JP, Lipponen JA, Ranta-Aho PO, Karjalainen PA. Kubios HRV--heart rate variability analysis software. Comput Methods Prog Biomed. 2014;113(1):210-20.

21. Heathers JA. Everything hertz: methodological issues in short-term frequency-domain HRV. Front Physiol. 2014;5:177.

22. Goldberger JJ, Le FK, Lahiri M, Kannankeril PJ, Ng J, Kadish AH. Assessment of parasympathetic reactivation after exercise. Am J Physiol Heart Circ Physiol. 2006;290(6):H2446-52.

23. Otzenberger H, Gronfier C, Simon C, Charloux A, Ehrhart J, Piquard F, Brandenberger $G$. Dynamic heart rate variability: a tool for exploring sympathovagal balance continuously during sleep in men. Am J Phys. 1998; 275(3 Pt 2):H946-50.

24. Reyes del Paso GA, Langewitz W, Mulder L, van Roon A, Duschek S. The utility of low frequency heart rate variability as an index of sympathetic cardiac tone: a review with emphasis on a reanalysis of previous studies. Psychophysiology. 2013;50(5):477-87.

25. Sztajzel J. Heart rate variability: a noninvasive electrocardiographic method to measure the autonomic nervous system. Swiss Med Wkly. 2004;134(3536):514-22.

26. Quintana DS. Statistical considerations for reporting and planning heart rate variability case-control studies. Psychophysiology. 2017;54(3):344-9.

27. Sondermeijer HP, van Marle AG, Kamen $\mathrm{P}$, Krum H. Acute effects of caffeine on heart rate variability. Am J Cardiol. 2002;90(8):906-7.

28. Zimmermann-Viehoff F, Thayer J, Koenig J, Herrmann C, Weber CS, Deter HC. Short-term effects of espresso coffee on heart rate variability and blood pressure in habitual and non-habitual coffee consumers-a randomized crossover study. Nutr Neurosci. 2016;19(4):169-75.

29. Gurley BJ, Steelman SC, Thomas SL. Multi-ingredient, caffeine-containing dietary supplements: history, safety, and efficacy. Clin Ther. 2015;37(2):275-301.

30. Koenig J, Jarczok MN, Kuhn W, Morsch K, Schäfer A, Hillecke TK, Thayer JF Impact of caffeine on heart rate variability: a systematic review. J Caffeine Res. 2013;3(1):22-37.

31. Rauh R, Burkert M, Siepmann M, Mueck-Weymann M. Acute effects of caffeine on heart rate variability in habitual caffeine consumers. Clin Physiol Funct Imaging. 2006;26(3):163-6.

32. Yoshinaga Costa JB, Gomes Anunciação P, Ruiz RJ, Casonatto J, Doederlein Polito M. Effect of caffeine intake on blood pressure and heart rate variability after a single bout of aerobic exercise. Int J Sports Med J. 2012; 13(3):109-21.

33. Graham T, Spriet L. Metabolic, catecholamine, and exercise performance responses to various doses of caffeine. J Appl Physiol. 1995;78(3):867-74.

34. Greer F, McLean C, Graham T. Caffeine, performance, and metabolism during repeated Wingate exercise tests. J Appl Physiol. 1998;85(4):1502-8.

35. Crowe MJ, Leicht AS, Spinks WL. Physiological and cognitive responses to caffeine during repeated, high-intensity exercise. Int I Sport Nutr Exerc Metab. 2006;16(5):528-44.

36. Xhyheri B, Manfrini $O$, Mazzolini M, Pizzi C, Bugiardini R. Heart rate variability today. Prog Cardiovasc Dis. 2012;55(3):321-31.

37. Haller CA, Duan M, Jacob P, Benowitz N. Human pharmacology of a performance-enhancing dietary supplement under resting and exercise conditions. Br J Clin Pharmacol. 2008;65(6):833-40.

38. Kliszczewicz BM, Esco MR, Quindry JC, Blessing DL, Oliver GD, Taylor KJ, Price BM. Autonomic responses to an acute bout of high-intensity body weight resistance exercise vs. treadmill running. J Strength Cond Res. 2016; 30(4):1050-8.

39. Reimann M, Rudiger H, Weiss N, Ziemssen T. Acute hyperlipidemia but not hyperhomocysteinemia impairs reflex regulation of the cardiovascular system. Atherosclerosis Supp. 2015;18:8-15.

Ready to submit your research? Choose BMC and benefit from:

- fast, convenient online submission

- thorough peer review by experienced researchers in your field

- rapid publication on acceptance

- support for research data, including large and complex data types

- gold Open Access which fosters wider collaboration and increased citations

- maximum visibility for your research: over $100 \mathrm{M}$ website views per year

At BMC, research is always in progress.

Learn more biomedcentral.com/submissions 